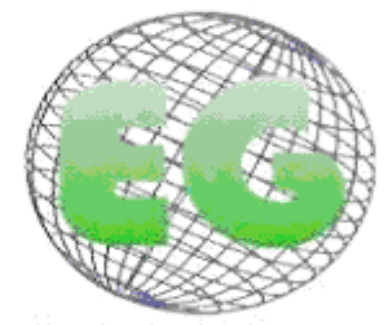

ISSN 1695-6141 N'25.

www.um.es/egloball

\title{
ENSAYOS
}

\section{Lepra. Impacto psicosocial.}

\author{
Leprosy. Psychosocial impact
}

\section{*Blanco Córdova, CA., **Cangas García, T.}

\author{
*Esp. I Grado Dermatología Pol. Docente "Dr. Isidro de Armas". Profesor Instructor. **Enfermera \\ Encuestadora. Programa ITS/VIH/SIDA. Pol. “ Dr. Isidro de Armas” . La Habana. Cuba.
}

Palabras clave: lepra; enfermedad de Hansen; bioética; humanismo.

Keywords: leprosy; Hansen' s illlness; bioethics; humanism.

\section{RESUMEN}

Las enfermedades infecciosas se han convertido en un importante problema de salud, y reto para científicos, médicos e investigadores. En la actualidad son cientos de miles de personas que padecen de Lepra. El estigma y las discapacidades producidas los convierten en inhabilitados sociales. La aparición de la Bioética como nuevo paradigma del pensamiento médico, con sus principios de beneficencia, no maleficencia, autonomía y justicia, ha conmocionado al mundo de las ciencias médicas.

Se realiza un análisis filosófico de un dilema bioético importante en nuestro medio: la ética médica y la Enfermedad de Hansen (Lepra), el humanismo como base de la relación médico paciente y la reflexión acerca del impacto psicosocial y económico de la Lepra. Se realizan algunas reflexiones que debe tener en cuenta el médico y enfermera de familia, así como el resto del Equipo Básico de Salud y los trabajadores de la Atención Primaria, con el paciente de Lepra.

Se concluye que la relación médico-paciente en enfermos de Lepra requiere un humanismo vivo y desde el punto de vista psicosocial, hay que vencer los tabúes y la discriminación que desata el padecer de Lepra en la comunidad.

\section{ABSTRACT}

Infectious illnesses are an important health problem and a challenge for scientists, doctors and investigators. At the present time there are hundreds of thousands of people suffering from leprosy. The stigma and the inabilities produced makes them socially disabled. The appearance of Bioethics as a new paradigm of medical thought, with its charity principles, non balefulness, autonomy and justice, has revolutionised the world of the medical sciences.

A philosophical analysis of an important dilemma ofbioethics in our environment is performed: the medical ethics and Hansen's illness (leprosy), humanism as the base of the patient- doctor relationship and the reflection in the impact psychosocial and economic impact of leprosy. Some reflections that doctors and nurses, as well as the rest of the Basic Health Team and Primary Attention workers should bear in mind when dealing with leprosy patients are offered. 
The conclusion is that the doctor-patient relationship when dealing with leprosy requires active humanism and, psychosocially, it is necessary to conquer the taboo and the discrimination that leprosy sparks off in the community.

\section{INTRODUCCIÓN}

La Lepra (Enfermedad de Hansen) es una enfermedad infecciosa crónica transmisible, propia del hombre, producida por el Mycobacterium Leprae, que afecta fundamentalmente la piel y el sistema nervioso periférico, donde en las formas graves existe compromiso de algunas vísceras y cuya exteriorización clínica polimorfa depende del comportamiento inmunológico del organismo. Esta enfermedad sigue siendo uno de los problemas de salud más graves en muchos lugares del mundo.

Tan antigua como el mismo hombre, aparece mencionada en los primeros escritos de civilizaciones antiguas, (año 2000 a.n.e.) en los libros de los Vedas y 300 años a.C., en el Susrutha Samhita con el nombre de Kustha. En China 500 años a.C. era conocida como La Feng. En Egipto se le denominaba Ucheda, y se ha comprobado en algunas momias y en el papiro de Ebers. También en el pueblo judío donde se cita en el Antiguo Testamento, denominada Zarahat. ${ }^{1,2}$

Causó verdaderos estragos a poblaciones enteras, azotando a todos los continentes. Ha sido considerada una enfermedad mutilante, incurable, repulsiva y estigmatizante, lo que ha generado un trato inhumano hacia las personas que la padecen, constituyendo aún hoy, un problema de salud importante para algunos países. ${ }^{2}$

Siempre fue identificada como un "castigo de Dios", "hijos del Dios del mal", como un "castigo divino", "una maldición", y fueron sometidos a vejaciones, privaciones de derechos, discriminaciones, persecuciones y acciones represivas. Estas creencias erradas respecto a la enfermedad, daban lugar a que las personas por temor a provocar la ira de los dioses y ser víctima de un castigo semejante, rechazaban, maltrataban y hasta mataban a estas personas. $^{3}$

Teniendo en cuenta que la medicina es una ciencia al servicio del hombre, la salud, más que un derecho, es una necesidad primordial del hombre y de la sociedad en su conjunto, y se convierte en un inestimable bien social. Las tareas de los servicios de salud deben ser prioridades del estado, a las que deben dedicarse todos los recursos. disponibles ${ }^{4}$

La Organización Mundial de la Salud (OMS) estima que los enormes progresos alcanzados en los tres últimos decenios en materia de desarrollo sanitario están en peligro, porque la desigualdad creciente entre ricos y pobres supone una amenaza sin precedentes para la salud y el bienestar de las poblaciones del globo (Nakajima, 1994). ${ }^{5}$

La profesión médica es casi tan antigua como la humanidad misma, surge de la necesidad de conjurar la enfermedad y la muerte. Es una continuación de la medicina preventiva, donde el médico era también "sacerdote" y "brujo", y ha sido objeto de cambios de carácter en los diferentes períodos históricos de acuerdo con la estructura de la sociedad en cada época y su concepción general del mundo. Por lo tanto, queda bien definida la condicionalidad psicosocial de la génesis de la medicina. ${ }^{1}$

¿Posee la actividad médica los atributos de la práctica socio-histórica? Por supuesto que sí. La medicina en su evolución histórica se ha comportado y se comporta en la unidad de los aspectos cognoscitivos, científico-experimentales, prácticos propiamente dicho y prácticos 
valorativos. La actividad médica es un producto histórico que tiene su origen en un momento específico, y adopta determinados rasgos en razón del carácter de la formación económico social por donde transita. ${ }^{6}$

En el 2008 enfermaron de Lepra en el mundo 249007 casos y de ellos, 41821 se diagnosticaron en nuestra América. El sudeste asiático también reportó una cifra aun superior de casos nuevos, ascendiendo a $167505.7,8$

Actualmente se diagnostican en Cuba entre 250 a 300 casos anuales, y pese a la voluntad política y social del estado, aún persiste el mismo rechazo social y discriminación, que tuvo la enfermedad desde sus comienzos. ${ }^{9}$

Tenemos una concepción científica del mundo, y los profesionales de la salud están preparados para dar respuesta a las necesidades sanitarias del pueblo, además, se tiene una concepción integral de la medicina y se percibe a la persona humana como una unidad biopsicosocioespiritual, ${ }^{10}$ por lo que el Ministerio de Salud Pública dedica sus esfuerzos y conocimientos a fomentar la salud física, psíquica y espiritual del hombre, a prevenir las enfermedades, a la atención al paciente y su rehabilitación.

Con apoyo en el hecho inobjetable de que la esencia del hombre es social, así como también lo es el proceso salud-enfermedad, el médico en nuestro medio se ocupa no sólo de los aspectos técnico-biológicos de la profesión, sino también de los psicosociales y ecológicos, por lo que la comunicación juega un rol importante en la relación médicopaciente, fundamentando el comportamiento del profesional de las ciencias médicas. Por todo lo anterior, creemos importante conocer y aplicar los aspectos bioéticos y psicosociales en el manejo del paciente enfermo de Lepra, por el médico y enfermera de la familia de su comunidad.

\section{Objetivo General:}

Caracterizar algunos aspectos bioéticos sociales en el manejo del paciente de Hansen, en la Atención Primaria de Salud (APS).

\section{Específicos:}

-Destacar la relación médico paciente como un factor muy importante en el manejo humanista del enfermo de Lepra.

-Determinar algunos aspectos psicosociales de la Lepra en la Atención Primaria de Salud.

\section{DESARROLLO:}

\section{ANÁLISIS Y DISCUSIÓN}

Nos enfocamos a realizar un análisis sobre la enfermedad de Hansen (Lepra) y su relación con aspectos tan importantes como la ética, el humanismo y bioética, así como el impacto de esta enfermedad en la sociedad y sus implicaciones psicosociales.

\section{Ética y lepra}

La ética, como rama del saber filosófico, hace su aparición mucho más tarde que la moral misma. En un principio, la ética, en cuanto a ciencia moral, no se había liberado aún del 
conocimiento filosófico, pero al convertirse en disciplina del saber filosófico, la ética deja de coincidir con la moral y ésta se convierte, por derecho propio, en un campo del saber filosófico, objeto de la ética (Organización Panamericana de la Salud, 1992). Por su parte la Filosofía, proviene del griego Phylos: amor, amigo o amante y Sophya: sabiduría, por lo que significa amigo de la sabiduría. La filosofía se originó, paralelamente con el deseo humano de satisfacer su curiosidad, de allí que sea tan vieja como el hombre y está indisolublemente relacionada con el planteamiento de cuestiones acerca de las cualidades fundamentales de la actividad humana.

La ética médica, como manifestación particular de la ética en general, trata específicamente sobre los principios y normas de conducta, que rigen el saber hacer profesional de los trabajadores de la salud. El problema más importante de la ética médica es la relación médico-paciente, e íntimamente ligado a ello la relación de los trabajadores de la salud entre sí y de éstos con los familiares del paciente. ${ }^{11}$ El objetivo es crear las condiciones óptimas, y por consiguiente, una terapia integral. ${ }^{12}$

Debemos Interpretar la relación médico-paciente como forma humana de comunicación en la cual se concretiza la relación individuo-sociedad. Es una relación social basada en un modelo de comunicación que en calidad de ley general de la actividad humana le confiere su base fisiológica y gnoseológica a los paradigmas vigentes en las ciencias médicas y debe comprenderse como una relación de comunicación que puede transcurrir bajo la forma Sujeto-Objeto, o bajo la forma Sujeto-Sujeto. En general, la práctica médica que se "reduce" a los fines de curar y rehabilitar, reserva al médico la función de sujeto y condena al paciente a obedecerlo.

Pero el modelo más avanzado de la medicina actual, al que nos encaminamos, además de curar y rehabilitar se propone prevenir la enfermedad y promover la salud, tiende a basarse en el modelo opuesto de comunicación, donde el médico y el paciente entran en relación recíproca como sujetos. Es decir, que la medicina preventiva y promocional, de énfasis social, se fundamenta en un modelo de comunicación Sujeto-Sujeto. ${ }^{13}$

La relación médico-paciente debe satisfacer la necesidad de seguridad que tiene el paciente, se le explica su estado de salud o los problemas inherentes a la enfermedad que padece, con la prudencia necesaria, y se informa oportunamente sobre las medidas diagnósticas y terapéuticas procedentes. Salvo en casos excepcionales, debe contarse con el consentimiento del paciente o los familiares cuando dichas medidas impliquen algún riesgo. Tanto una buena entrevista clínica y psicosocial, como un correcto examen físico, son elementos que permiten estrechar y hacer adecuada la relación médico-paciente. Por otro lado, debe tenerse en cuenta que cada una de las maniobras realizadas son objeto de evaluación por el paciente y comparadas con exámenes médicos previos.

En numerosas circunstancias, sobre la base de la experiencia cubana de trabajo con personas que padecen de Lepra, este diagnóstico ha revelado rechazo, aislamiento, tabúes de todo tipo, y se estigmatiza aun en muchos lugares hasta por familiares y amigos. El trato inhumano va en contra de algunos principios éticos, los cuales comenzaron a tomar fuerza con Van Rensselaer Potter, medico Oncólogo y profesor de la Universidad Médica de Winconsin. ${ }^{13}$

Al crear un ambiente de confianza y de respeto a la inviolable dignidad humana del paciente, se pueden conocer sus antecedentes, se estudia su personalidad, sus intereses, sus demandas, así como el estado de ánimo del sujeto en las condiciones evolutivas en que se encuentra su afección crónica, que hoy en día es curable. 
Es necesaria la aplicación de los principios éticos sustentados en la autonomía, en cuyo contexto el respeto irrestricto a la persona humana significa proteger a aquellos que puedan sufrir una pérdida de la propia decisión, a la vez que implica impartir conocimientos al individuo para que este tome sus decisiones y no surjan relaciones de dependencia con el médico. Es de señalar que muchos problemas de valores no conformados, descansan en profundas incomunicaciones, por lo que el médico está llamado a ser un buen comunicador. La naturaleza social del hombre hace de la comunicación humana, una condición indispensable del trabajo, del conocimiento y de la creación de sistemas de valores.

Otro principio, el de justicia, que se refiere a la existencia de equidad universal, para el sujeto significa principalmente la ausencia de discriminación e igualdad de oportunidades para el acceso a recursos y las cargas de riesgos y beneficios. Las sociedades también tiene obligación moral de facilitar un acceso igualitario a los servicios de salud y además, todo individuo tiene derecho moral a acceder a ellos. Es por ello que algunos gobiernos han incluido en su constitución y en otros códigos, disposiciones legales destinadas a cumplir con la obligación moral de brindar salud a todos sus asociados, siendo nuestro país uno de los que tiene establecido en su constitución, el derecho a la asistencia médica.

Estos pacientes tienen los mismos derechos y esperan que se les brinden siempre una atención concienzuda, diligente y conforme al estado de los conocimientos médicos. Esta concepción de la justicia en materia de salud parte de reconocerla como un derecho humano, institucionalizado por la sociedad, reconocido en un marco político y jurídico, y organizado de forma en que su práctica y sus servicios permitan el acceso a la población que usufructa los mismos.

El principio de la beneficencia, que implica la protección a las personas y se procura su bienestar. ${ }^{13}$ Ya desde Hipócrates, en su juramento de honor, condicionó la práctica del arte de la medicina, al beneficio de los pacientes, lo que es considerada por muchos la norma ética fundamental. Beanchamps y Children (1989) describen un principio más, la nomaleficencia, que se entiende como no causar daño o mal al paciente. ${ }^{14}$

\section{El humanismo y la bioética.}

En las últimas 5 décadas, el desarrollo de la medicina cubana ha constituido prioridad fundamental del Estado. No se trata solo de sus avances científicos y tecnológicos, sino en la conciencia y estructura de valores, desde los cuales se forman a los profesionales de la salud. De ahí que el sentido de la responsabilidad social profesional, se distinga por un profundo humanismo y una alta capacidad de respuesta frente a las necesidades sociales. Es decir, no se trata solo que se construyera la infraestructura científico-técnica necesaria para el desarrollo de la sociedad, sino que se forjó un profesional con una actitud nueva que ha continuado cultivándose aún en condiciones materiales difíciles. Los sistemas de salud deben proteger al paciente de la soledad, dado que la enfermedad y el dolor tienden a aislarlo; por esto, el sistema cubano contribuye a que estos pacientes afectados por la Lepra, asuman un estilo de vida que les permita encontrarse a sí mismos, de manera que, a pesar de su afección, pueda decirse que han alcanzado un desarrollo de su personalidad, en la que han encontrado su razón de ser o el sentido de su vida, o al menos, debe ponerlo en el camino de ese encuentro. Ser capaces, a través de la relación médico- paciente, descubrir la fuerza interior que moviliza al individuo, su riqueza espiritual, su amor por la vida, aplicar la ética adecuadamente, que fortalezca el cuerpo y que enriquezca la mente. Esto conlleva a una idea de contenido humanista de lo que debería ser un médico, o mejor aún, un profesional de la salud. ${ }^{15}$ 
Es importante que el profesional de la salud esté dispuesto a negociar con su enfermo a respetar sus puntos de vista, a aceptarlo como socio, como un colaborador en la empresa de buscar curación o alivio. Para que podamos inspirar "amor a la vida" y "respeto a las decisiones de los demás", es necesario, en primer lugar, que nosotros cultivemos ese amor y ese respeto; es necesario, en segundo lugar, una comunicación libre de barreras, que no quiere decir una transmisión de toda la verdad, sino una comunicación entre humanos, permeada por la verdadera empatía y la transmisión honesta del mensaje, "...no estás solo, estamos contigo, te aceptamos tal y cual eres". ${ }^{16}$ Así como el personal de salud debe necesariamente garantizar un elevado nivel científico-técnico y cultural, si descuida su desarrollo estético estará notablemente limitado en su proyección humanística, en su ejercicio ético, en la relación médico-paciente-familia-comunidad y en sus potencialidades para mitigar los sufrimientos implícitos en la pérdida de la salud de sus pacientes. ${ }^{17}$

El humanismo que emana de las teorías éticofilosóficas y corrientes avanzadas, tiene cierto sentido progresista: la expresión de la protesta contra el avasallamiento de la personalidad como defensa de los derechos y valores de la persona humana, desde el renacimiento europeo, que distinguía al hombre no por su origen, sino por sus méritos personales. ${ }^{11}$ Por otra parte, se tiene en cuenta el hecho indiscutible de que la salud de los profesionales corre riesgo como consecuencia de su trabajo. ${ }^{1}$ La relación que se establece entre el médico y enfermera con el paciente requiere de un humanismo vivo, que abarca una serie de matices que determinan la enfermedad; contexto donde el segundo sufre y la orientación que el médico sigue en el tratamiento es definitoria. Es la entrevista en el consultorio médico, el eje principal de la relación médico-paciente.

El médico y la enfermera frente a un paciente con Lepra deben romper, ante todo, un muro a veces muy sólido, que de no hacerlo pudiera aislarlo para siempre del paciente, si se tiene en cuenta que este siente, por lo general, temor a la muerte, angustia ante el futuro y lo desconocido, tristeza y hostilidad ante el infortunio y una permanente demanda de afecto. ${ }^{18}$

Nuestra tarea suprema de la educación médica es la humanización del hombre, la apropiación de los valores, de las costumbres, de las tradiciones, que en última instancia se produce por medio de la aprehensión de la cultura nacional y universal, para de esta manera romper mitos y leyendas sobre enfermedades marginadas hasta nuestros días como es el caso de la Lepra. De igual manera, no puede haber proyecto social sin una riqueza individual extrema.

El diagnóstico de Lepra sólo debe y puede interpretarse de esta forma: que la persona requiere consejos profesionales respecto a la infección y a la enfermedad, chequeos periódicos, seguimientos posteriores en el periodo de observación antes del alta y, sobre todo, una buena educación para continuar viviendo con su afección de base y cooperar para curarse definitivamente o mitigar las discapacidades que pudiera dejar como secuelas, ¿Cómo proceder para que el impacto negativo o sus consecuencias afecten lo menos posible su propio bienestar y el de las personas que la rodean? Un diagnóstico positivo no implica ahora la muerte como ocurría en el siglo pasado, ni la terminación de una vida normal, pero sí impone la necesidad de ordenar prioridades y cuidados, especialmente los que se refieren a las medidas para evitar la transmisión. Sí se conoce que por el hecho de padecer la enfermedad y llevar el tratamiento indicado, el paciente deja de transmitir los bacilos y puede llevar una vida normal, además de curarse definitivamente.

Se plantea por la OMS/OPS en su nueva estrategia para eliminar la Lepra en el 2015 como un problema de salud en el mundo, mantener los principios de la sostenibilidad, la calidad de los servicios que se le brinda a estos pacientes, de la equidad y la accesibilidad, así como 
de temas relacionados con los derechos humanos, existiendo el compromiso de mantener una prevalencia menor de $1 \times 10000$ habitantes, como lo acordado por la OMS en el año 1991. ${ }^{19}$ Se trabaja para cumplir la meta de reducir la carga económica y social de la Lepra en la región de las Américas, asegurando el tratamiento completo y oportuno a los casos enfermos así como la introducción de la quimioprofilaxis a todos los contactos. También reducir la tasa de discapacidad grado II, incrementar la cobertura del examen al $100 \%$ de los contactos del caso índice.

Aunque la Bioética aparece como un nuevo paradigma social y cultural consistente en la confrontación de hechos y valores en la esfera de las ciencias de la vida y la atención sanitaria, debemos tener en cuenta que los conflictos de valor de la medicina contemporánea han obligado a reevaluar el valor de la vida humana y su dignidad, sabiendo que sin justicia social, los intentos que se hagan en aras de humanizar la atención sanitaria a la larga fracasarán. ${ }^{20}$

\section{Impacto de la lepra en la sociedad. Aspectos psicosociales.}

La psicología social es el reflejo directo del modo de vida del ser de la conciencia de las masas, o sea la integran las concepciones e ideas que se plasman en el curso de la vida y la actividad cotidiana de las masas y que reflejan las condiciones de vida y actividad, los intereses y necesidades de estos.

La Lepra es una enfermedad estigmatizada por la sociedad, que se debe, fundamentalmente, al impacto social que produce la misma. Es por ello que se considera una enfermedad psicosocial, económica, política y cultural.

Es una de las primeras causas de incapacidades y mutilaciones. Se estima que el $25 \%$ de los enfermos que no son detectados y tratados tempranamente, desarrollan insensibilidad y/o severas deformidades de manos y pies ${ }^{21}$, lo que constituye una seria desventaja en la vida diaria.

Esta enfermedad también afecta la vista, pero la frecuencia de las complicaciones oculares varía de un país a otro. Entre las enfermedades transmisibles, la Lepra, se considera como la mayor causa de ceguera junto con el Tracoma y Oncocercosis. ${ }^{22}$

No sólo afecta al paciente, a familiares y amigos, sino que ha demostrado tener efectos considerables sobre toda la sociedad. Sus consecuencias han repercutido sobre instituciones religiosas, políticas y sociales, en fin, sobre la forma de vivir, y nos ha obligado tanto a reflexionar, como a evaluar conductas que permitan enfrentar con éxito la enfermedad.

Es necesario comprender el papel que juega la familia como elemento muy importante en la atención y cuidado de estas personas afectadas por la enfermedad de Hansen o Lepra. Este papel no podrá ser nunca sustituido por persona alguna, ya que, como se sabe, es quien más involucrada está desde el punto de vista emocional, legal y ético. La familia es quien más tiempo está con el enfermo, y por lo tanto, es la que debe llevar a cabo los cuidados de la manera más integral posible. ${ }^{23}$

La familia, al igual que el paciente, pasa por las distintas fases de la enfermedad, sufre como si estuviera enfermo, se crean situaciones de ansiedad, frustración, resentimiento e insatisfacción. 
La Lepra tiene una repercusión social, humanitaria y de principios legales. Desde el punto de vista de la sociedad, uno de los grandes problemas que deben vencerse es la discriminación que desata esta enfermedad, en tanto los afectados no sólo siguen sometidos al riesgo de las deformidades que los incapacitan para el trabajo, sino también a la inhabilitación social que deriva del rechazo de que son objetos. Debemos recordar que la teoría marxista ha demostrado que la conciencia social no es más que un reflejo del ser social.

Esta enfermedad es una dolencia peculiar por el riesgo real de discriminación que provoca en las personas portadoras del Mycobacterium Leprae. Es por este motivo que se valoran tanto los principios de la confidencialidad y privacidad, porque una vez quebrados, exponen a los pacientes a serios riesgos de discriminación. ${ }^{24,25}$

Los trabajadores de la salud deben tener en cuenta en su relación con los pacientes estos valores éticos. Las características del médico en esta interrelación, las resumió Hipócrates hace más de 2000 años cuando consideró que el médico debía reunir 4 cualidades fundamentales: conocimientos, sabiduría, humanidad y probidad.

Resulta necesario utilizar todos los medios para desarrollar en los miembros del equipo de salud y en sus usuarios, la conciencia plena de que el humanismo, la espiritualidad, y los comportamientos éticos en las relaciones profesionales, exigen una interacción humana recíprocamente respetuosa, afable, de empatía y cortés. ${ }^{26}$

En la actualidad, en la atención médica se puede mencionar como negativo la pobre relación médico-paciente, la información inadecuada a pacientes y familiares, cometer indiscreciones, violaciones del respeto al pudor y violaciones de la educación formal y de la disciplina, con una pobre actitud crítica y autocrítica. Precisamente esas deficiencias subjetivas plantean un reto para la acción. ${ }^{27}$

Si somos capaces de superar el conflicto ético, será una muestra inequívoca de generosidad y de humanismo ofrendada a la sociedad. La sensibilidad humana, la preocupación real por el otro, es una condición del desarrollo de valores más complejos, necesarios para enfrentar y erradicar esta enfermedad.

Hemos podido llegar a convivir y aceptar a la pandemia del VIH/SIDA, en solo unos años y sin embargo, aun en nuestros días somos incapaces de aceptar a la Lepra, enfermedad tan milenaria como el mismo surgimiento del hombre.

El temor al contagio por parte de la comunidad cercana es la consecuencia psicosocial más terrible de la infección por Lepra y afecta tanto el sistema de apoyo familiar, como el entorno del paciente. Los múltiples mitos que se han tejido a través de los siglos alrededor de esta enfermedad, aun persisten en la actualidad y entorpecen su manejo en las diferentes áreas de salud de Cuba y del mundo. ${ }^{28}$ Es por ello que los profesionales de la salud en particular y la sociedad en general, están llamados a realizar una actividad transformadora en el desarrollo del proceso salud-enfermedad, capaces de conocer y actuar en la preservación y recuperación de la salud humana, tanto individual como colectiva.

Desde el punto de vista económico, el impacto es enorme, porque la mayoría de las personas afectadas se localizan en el grupo de edad más productiva (20-40 años). Esto es especialmente significativo, si se tiene en cuenta que muchos países atraviesan una grave crisis económica, que a su vez limita la posibilidad de importación de medicamentos y otros suministros para combatir la enfermedad (personal preparado en el tratamiento y 
seguimiento del paciente, educación sanitaria, rehabilitación de discapacidades, exámenes de laboratorio, etc.) ${ }^{29}$

Es importante destacar que la enfermedad no limita, por sí misma, la capacidad de trabajo, por lo que se torna una capacidad básica de solidaridad humana buscar las condiciones adecuadas para que las personas infectadas puedan continuar sus tareas habituales. Solo las discapacidades producidas por diagnósticos tardíos, son las causantes de limitaciones físicas, psíquicas y laborales, que de no ser corregidas a tiempo pudiera llevar a estos pacientes a la marginalidad, exclusión social, minusvalía y mendicidad. ${ }^{30,31}$ También puede impactar seriamente a la mujer y la afecta en su función de madre, esposa, educadora, sostén económico, depositaría de la cohesión familiar. En este sentido, pensamos que proteger a la mujer implica proteger a toda la familia, y por ende, a la sociedad.

La Lepra y la niñez es un aspecto sensible sobre todo en países de bajo desarrollo socioeconómico y condiciones de hacinamiento como África y Asia. En Cuba en el año 1970, el número de casos en menores de 15 años estaba alrededor de 20 niños anuales afectados, y esto ha ido bajando a medida que el sistema de salud se ha ido fortaleciendo y desarrollando, sumado a la entrada de nuevas drogas en el tratamiento de esta enfermedad que ya logran su cura total. Luego del año 2000 la cifra de niños en nuestro país es mucho menor y solo se reportan entre 5 a 10 casos anuales. ${ }^{32}$

La estadísticas mundiales del 2008 apuntan que hasta el $6 \%$ de los casos infestados son menores de 15 años. ${ }^{33}$ ¿hasta dónde los padres son responsables?, ¿hasta dónde el personal médico? Por supuesto que el rechazo trae consigo problema psicosociales propios de los pacientes minusválidos, pues la percepción propia de estos pacientes, los hace sentirse sensiblemente diferentes a los demás, lo que trae aparejado depresión, aislamiento y agresividad. Además existe la percepción referencial, o sea la percepción que el propio paciente intuye que los demás tienen de él, y al parecer y según estudios, a esta última le dan más importancia que a la percepción primera. ${ }^{34,35}$

Pensamos que el médico y el psicólogo desempeñan una función vital en la orientación y persuasión científica y humana sobre las cuestiones preventivas y curativas de la enfermedad. Si nos comportáramos humanamente con los pacientes de Lepra, estos problemas sicológicos se minimizarían al máximo y no se sentirían unos inadaptados sociales. Todos los esfuerzos realizados son pocos en lo relacionado con el afán incesante por cumplir al pie de la letra las actividades de control y tratamiento de esta enfermedad y otros problemas específicos, con la visión integradora de la salud en los procesos de desarrollo. Entre los trabajadores de la salud, el paciente, la familia y la sociedad, está el núcleo fundamental para la conducción de estas personas. Acercarnos a ellos con cariño, amabilidad y esperanza, es nuestra principal función. ${ }^{36}$

Por todo lo anteriormente expuesto se debe resaltar que el enfoque bioético en la atención del paciente de Lepra en nuestro sistema de salud es, además de jurídica, una cuestión humana y social, a diferencia de los sistemas neoliberales en los que existe la deshumanización económica. La instrucción de la sociedad en temas relacionados con esta enfermedad, a los trabajadores de la Atención Primaria, junto a todo el sistema de salud, permitirá la reinserción laboral y social adecuada de estos pacientes. ${ }^{37}$

\section{CONCLUSIONES}

- La relación médico-paciente en enfermos de Lepra requiere un humanismo vivo. 
- $\quad$ Desde el punto de vista psicosocial, hay que vencer los tabúes y la discriminación que desata el padecer de Lepra en la comunidad.

\section{RECOMENDACIONES}

Sabemos que la Lepra se cura. No obstante, para detener la cadena epidemiológica y erradicarla de nuestro país, es esencial la aplicación consecuente del Programa Nacional para el Control de la Lepra en Cuba, en las aéreas de atención primaria de salud como eslabón principal (médico y enfermera de la familia), así como llevar al resto del Grupo Básico de Trabajo estas reflexiones sobre aspectos bioéticos de la Lepra.

\section{BIBLIOGRAFÍA}

1-Terencio de las Aguas J. Consideraciones histórico-epidemiológicas de la lepra en América. Med Cutan Iber Lat Am Alicante, España. 2006; 34:179-194.

2-Rivero Reyes Eduardo, Barrios Martínez Zoila, Berdasquera Corcho Denis, Tápanes Fernández Thelma, Peñalver Sinchay Ana Gladys. La lepra, un problema de salud global. Rev Cubana Med Gen Integr [revista en la Internet]. 2009 Mar [citado 2011 Feb 09]; 25(1): Disponible en: $\quad$ http://scielo.sld.cu/scielo.php?script=sci arttext\&pid=S086421252009000100010\&lng=es

3-González Prendes MA. Historia de la lepra en Cuba. La Habana: Empresa Consolidada de Artes Gráficas; 1963

4-Gabinete Central Docente Metodológico. Edición de 1979: Introducción a la especialidad. La Habana: Instituto Superior de Ciencias Médicas de la Habana, 1979. 11-8 (impresión ligera).

5-Nakajima, H. La creciente desigualdad: una cuestión de vida o muerte. Salud Mundial. 1994: 47 (6), 3-4.

6-Mayoral Olazábal Mercedes, Bujardón Mendoza Alberto, Flores Rodríguez Jorge L.: "El diagnostico médico y los métodos del conocimiento científico", Proyecto Editorial CENDECSA, Universidad Médica Carlos J. Finlay, Camagüey, Cuba, 1999.

7-Organización Mundial de la Salud. Lepra. Febrero de 2010. OMS. [Revisado 25 Febr 2011] Disponible en: http://www.paho.org/Spanish/AD/DPC/CD/lep-sit-reg-2007.pdf

8-Organización Mundial de la Salud. Lepra (Enfermedad de Hansen). Consejo ejecutivo

OMS, 126 reunión. Punto 4.21 del orden del día. EB 126/41. 22 de Enero del 2010.

9-Ministerio de Salud Pública. Programa Nacional para el Control de la Lepra en Cuba.

MINSAP. 2002, Ed. Rev. La Habana, Cuba.

10-Dueñas, J, R. Fuillerat, N. Pérez: La unidad cuerpo, mente y espíritu: una reflexión necesaria. La Habana: VI Encuentro Latinoamericano de Psicoanalistas y Psicólogos Cubanos (tema libre), 1996.

11-Alonso, D.: Ética y Deontología Médica, 1ra. ed. La Habana: Editorial Ministerio de Salud Pública, 1979; 1-10.

12-Acosta, J.: Vivir y morir con dignidad. La Habana: Hospital-Facultad "Calixto García." 2 (impresión ligera). 1996.

13-Pico, MV, MT Delgado y R Altisent: Aspectos éticos y legales de la investigación en Atención Primaria. Rev Méd de Aten Prim. 1995, 2 (4), 212-9.

14-Beauchamp, TL, FJ.:Children. Principles of Biomedical Ethics. New York: Oxford University Press. 1989: 34

15-Martínez Suárez N. Política, ciencia, ética y sociedad: su conjunción dialéctica en la experiencia del Contingente Henry Reeve. Revista Humanidades Médicas. 2006; 18(6). [consultado el 12 de diciembre de 2008]. Disponible en: 
http://scielo.sld.cu/scielo.php?script=sci arttext\&pid=S1727-

$81202006000300004 \& \operatorname{lng}=e s \& n r m=i s o \& \operatorname{lng}=e s$

16-González Pérez Camilo, Durán Morales Tatiana, Casals Blet Idalmis, Lugones Botell Miguel, Castro Rojas Tania. Reflexiones en torno a los problemas éticos y bioéticos en el tratamiento del paciente con VIH/SIDA. Trabajos de Revisión. Revista Cubana de Medicina General Integral. 2009; 25(3):105-112

17-Lorenzo Felipe D, López Domínguez M, Vinarde Lorenzo JM. Pensamiento médico desde una perspectiva estética. Correo Científico Médico de Holguín. 2006; 10(4).

[consultado el 12 diciembre de 2008]. Disponible en:

http://www.cocmed.sld.cu/no104/n104cbrev1.htm

18-Dueñas, J, N. Pérez. Psicología de la Salud: letra y espíritu. Rev. Cub. Psicol. 2003: 20 (1), 67-70. (En imprenta)

19-Organización Mundial de la Salud (OMS). Weekly epidemiological record. Relevé épidémiologique hebdomadaire 27 august 2010, 85th year, No. 35, 2010, 85, 337-348. [Revisado Enero 2011] Disponible en: http://www.who.int/wer

20-García Capote, J. Notas de clases de Curso de Filosofía. La Medicina contemporánea y sus principales dilemas. Facultad de Ciencias Médicas Girón. La Habana, 2011.

21-Organización Panamericana de Salud. La lepra en las Américas. Boletín Epidemiológico de la OPS, 1983. Vol. 4, No. 6.

22-Lechat, M.: Leprosy in a Global Context. 5th International Workshop on Leprosy Control in Asia. 1983.

23-Fernández Sacasas JA. Aspectos éticos de la educación médica: apuntes para una discusión de grupo. Tarija, Bolivia; 2001.p.47-64

24-Walker SL, Lockwood DN. Leprosy. Clin Dermatol. Mar-Apr 2007; 25 (2):165- 72.

25- Manzur Katrib J, Díaz Almeida JG, Cortes Hernández M, Ortiz González Pedro R, Sagaró Delgado B, Abreu Daniel A. Dermatología. Cuidad de La Habana: Ciencias Médicas; 2002.

26-González Menéndez R. Humanismo y gestión de salud: pasado, presente y futuro. Rev Cubana Salud Pública. 2006; 32(4). [consultado el 9 de enero de 2010]. Disponible en:http://scielo.sld.cu/scielo.php?script=sci arttext\&pid=S0864-

34662006000400006\&lng=es\&nrm=iso\&tlng=es

27-Llorens Figueroa JA. Ética y educación en los servicios de salud. Rev Cubana Salud

Pública. 2006; 32 (4). [consultado el 9 de enero de 2010]. Disponible en:

http://scielo.sld.cu/scielo.php?script=sci arttext\&pid=S0864-

$34662006000400013 \& \operatorname{lng}=e s \& n r m=i s o \& \operatorname{lng}=e s$

28-MedlinePlus. Información de salud para usted. Lepra, 2009. [consultado 6 Sep 2010). Disponible en:

http://www.nlm.nih.gov/medlineplus/spanish/ency/article/001347.htm

29-Suarez Moreno, Odelaisy. Baciloscopía de la Lepra. Material docente del Instituto de Medicina Tropical "Pedro Kouri" (IPK) Ciudad Habana, Cuba, 2007.

30-IPK. Enfermedades transmisibles e infecciosas: Lepra. Febrero del 2010. Disponible en: http://www.ipk.sld.cu/indice.htm. Tomado y adaptado de: Organización Mundial de la Salud 2010, [consultado 23 Mar 2010]. Disponible en: http://www.who.int/mediacentre/factsheets/fs101/es/index.html

31-Suárez Moreno Odelaisy, Hernández Gutiérrez Olenia, Hurtado Gascón Laura, Alonso Gómez María Elena, Pesant Hernández Olenia, Abab Machado Adis. Diagnóstico de la Lepra: Manual práctico para MGI. Instituto de Medicina Tropical "Pedro Kourí" (IPK), Ciudad de La Habana, 2007.

32-S. Paredes, M. Recarte, A. Albertengo, J. Monti. Lepra infantil. Rev Intern Dermatol Dermocosm 2002; 5: 348-351.

33-Scott Smith Darvin, Ramachandra Tara. eMedicine. Leprosy. Aug 19, 2008. [cited 2009 Jul 9]. Disponible en: http://emedicine.medscape.com/article/220455-overview 34-Oramas 
Fernández Diana Karina, Cruzata Rodríguez Larixa, Sotto Vargas Juan David, Poulot Limonta Santos. Comportamiento de la lepra en dos policlínicos del municipio La Lisa. Rev Cubana Med Gen Integr [revista en la Internet]. 2009 Dic[citado 2011 Feb 07]; 25(4): 7785. Disponible en:

http://scielo.sld.cu/scielo.php?script=sci arttext\&pid=S0864-1252009000400008\&lng=es. 35-Enciclopedia Cubana. EcuRED. Lepra enfermedad de Hansen. Enero de 2011 [citado el 9 feb 2011]. Disponible en: http://www.ecured.cu/index.php/Lepra enfermedad de hansen [Citado 30 Enero 2011] Tomado de: http://es.wikipedia.org/wiki/Lepra

36-Anderson H, Stryjewska B, Boyanton BL, et al. Hansen disease in the United States in the 21st century: a review of the literature. Arch Pathol Lab Med. Jun 2007; 131(6):982-6.

37-Van Veen NH, Nicholls PG, Smith WC, Richardus JH. Corticosteroids for treating nerve damage in leprosy. Cochrane Database Syst Rev. Apr 18, 2007; CD005491. 